\title{
Design of a channel drop filter by using a donor-type cavity with high- quality factor in a two-dimensional photonic crystal slab
}

\section{$\operatorname{AUTHOR}(\mathrm{S}):$}

Akahane, Y; Mochizuki, M; Asano, T; Tanaka, Y; Noda, S

\section{CITATION:}

Akahane, Y ... [et al]. Design of a channel drop filter by using a donor-type cavity with highquality factor in a two-dimensional photonic crystal slab. APPLIED PHYSICS LETTERS 2003, 82(9): 1341-1343

\section{ISSUE DATE:}

2003-03-03

URL:

http://hdl.handle.net/2433/50217

\section{RIGHT:}

Copyright 2003 American Institute of Physics. This article may be downloaded for personal use only. Any other use requires prior permission of the author and the American Institute of Physics. 


\title{
Design of a channel drop filter by using a donor-type cavity with high-quality factor in a two-dimensional photonic crystal slab
}

\author{
Yoshihiro Akahane, ${ }^{\text {a) }}$ Masamitsu Mochizuki, Takashi Asano, Yoshinori Tanaka, \\ and Susumu Noda \\ Department of Electronic Science and Engineering, Kyoto University, Core Research for Evolutional \\ Science and Technology (CREST), Japan Science and Technology Corporation (JST), Kyoto 606-8501, Japan
}

(Received 31 July 2002; accepted 4 January 2003)

\begin{abstract}
We report a design of the surface-emitting-type channel drop filters based on point defect cavities and line defect waveguides in two-dimensional photonic crystal slabs, which aim to improve the filtering resolution and light emission characteristics. Since the filters are passive, the mode volume size of the defects needs not be minimized, but the interaction between the defect cavity and the line defect waveguide must be considered. By adopting a donor-type point defect with three missing holes of linear shape, the quality factor of the filter theoretically increases to values as high as 2900 while it reached only 500 in the previously utilized acceptor-type defect. The results suggest that this donor-type defect is very useful for the development of ultrasmall channel add/drop devices. (C) 2003 American Institute of Physics. [DOI: 10.1063/1.1556556]
\end{abstract}

A two-dimensional (2D) photonic crystal (PC) slab- ${ }^{1-9}$ a thin dielectric slab with a $2 \mathrm{D}$ photonic crystal pattern-has attracted much attention as a relatively easy method to achieve a gap in the photonic mode spectrum. Recently, we have reported a very interesting phenomenon in which photons propagating along a line defect (waveguide) are trapped and emitted to the free space by a single point defect created in an air bridged 2D PC slab with triangular lattice patterns of air holes, as shown in Fig. 1(a). ${ }^{1}$ Moreover, we have shown that the tuning of emission wavelength is possible by selecting appropriate geometry of the structure while maintaining the maximal emission efficiency. ${ }^{3,4}$ The phenomena can be applied for surface-emitting-type ultrasmall channel add/drop filters for wavelength division multiplexed (WDM) optical communication systems.

Such application requires considerably high device performance such as high filtering resolution and coupling efficiency with external optics. Our past studies have focused on devices which utilize acceptor-type defects (enlarged air hole rods). However, the quality $(Q)$ factors of those filters, which determine the resolution of the filtering operation, are at most 500, and are insufficient for the application to WDM systems. Meanwhile, over the past few years, several studies have been done by other groups on the improvement of $Q$ factors of defect cavities. ${ }^{8,9}$ In such studies, the combination of the high $Q$ factors and small mode volumes are considered very important since the goal is to realize high performance active light-emitting devices such as zero-threshold lasers, etc. However, the requirement for the mode volume size is not essential for this channel add/drop filter since it is a passive device, as long as the cavity is single mode for the concerning spectral range. (Free spectral range more than 30 $\mathrm{nm}$ is required for WDM applications.) Instead, we must consider the interaction between the defect cavity and the line defect waveguide.

${ }^{a)}$ On leave from: Itami Research Laboratories, Sumitomo Electric Industries, Ltd., Itami, Hyogo 664-0016, Japan; electronic mail: y-akahane@ sei.co.jp
The $Q$ factor depends on the magnitude of coupling between the defect mode and the external environments, which are the line defect waveguide and the free space in the case of our device, since the photons are confined completely by the photonic band gap effect in the in-plane direction. The total $Q$ factor can be expressed as

$$
1 / Q=1 / Q_{\text {in }}+1 / Q_{\mathrm{v}},
$$

where $Q_{\text {in }}\left(Q_{\mathrm{v}}\right)$ represents a quality factor evaluated by assuming the coupling loss only to the waveguide modes (to the free-space modes). ${ }^{3,5}$ Although we can limitlessly increase $Q_{\text {in }}$ by increasing the distance between the defect and the waveguide, we should note that $Q_{\mathrm{v}}$ must be equal to $Q_{\text {in }}$ in order to obtain the maximum output efficiency of the filtering device (which is 50\%). ${ }^{3,10}$ Therefore, the total $Q$ becomes half of $Q_{\mathrm{v}}$ when the output efficiency is optimized to become maximum, so that high $Q$ filters need high $Q_{\mathrm{v}}$ cavities.

In this letter, we investigate various donor defects with one to three missing holes which are filled with the same dielectric substance as the slab. From now on, we call the

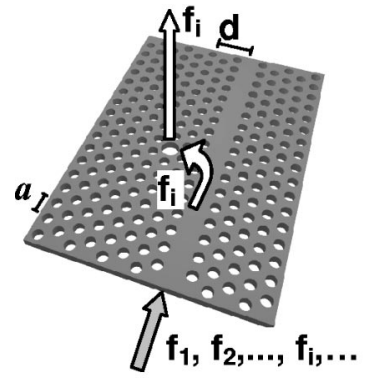

(a)

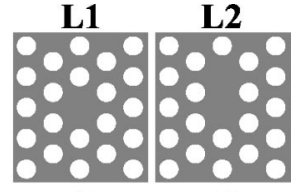

(b)

(c)

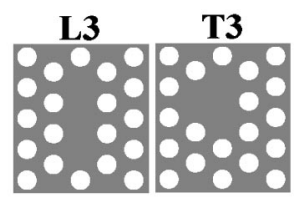

(d)

(e)
FIG. 1. (a) Schematic of a surface emitting channel add/drop filtering device, which consists of $2 \mathrm{D}$ PC slab with a line defect waveguide and a single point defect. The definitions of lattice constant $a$ and the distance $d$ between the waveguide and the defect are also shown. (b)-(e) Schematics showing the definitions of defect types. 
TABLE I. Quality factors and resonant frequencies of isolated defect cavities calculated by 3D-FDTD method. The defect types are defined in Figs. 1(b)-1(e). The quality factors are for the fundamental mode, and not for the next higher order mode. The table also includes the results for the acceptor type defect (A1) with a typical air hole radius $(0.56 a)$ and transmitting range of the waveguide which is taken from the Ref. 3.

\begin{tabular}{cccc}
\hline \hline & $\begin{array}{c}\text { Quality factor } \\
\text { Defect type }\end{array}$ & \multicolumn{2}{c}{ Frequency $(c / a)$} \\
\cline { 3 - 4 } & (fundamental mode) & Fundamental mode & Next higher mode \\
\hline L1 & 300 & 0.288 & 0.288 \\
L2 & 1400 & 0.273 & 0.288 \\
L3 & 5200 & 0.267 & 0.285 \\
T3 & 2500 & 0.267 & 0.278 \\
A1 & 900 & 0.274 & $\ldots$ \\
Waveguide & $\cdots$ & $0.263-0.280$ & $\ldots$ \\
& & (transmitting range) \\
\hline
\end{tabular}

defects shown in Figs. 1(b)-1(e) as L1 (one missing air hole), L2 (two missing air holes), L3 (three missing air holes of a linear shape), and T3 (three missing air holes of a triangular shape), respectively. We discuss no defect with asymmetric shape because it is not promising from the viewpoint of the spatial quality of the output light. The spatial and polarization modes of the light emitted to free space are also important which determine the coupling efficiency with external optics. We present a theoretical analysis on the frequency, quality factor, and light-emission characteristics of the defects.

As the first step in our analysis, we investigated isolated defects, i.e., single defects in the PC slab without waveguides, and evaluated the $Q$ factors and resonant frequencies of the defect cavities. In this case, the total $Q$ of an isolated defect becomes equal to $Q_{\mathrm{v}}$ since $Q_{\text {in }}$ becomes infinite [see Eq. (1)]. We used the three-dimensional (3D) finitedifference time-domain (FDTD) method ${ }^{11,12}$ for the calculation and assumed that a radius of air holes and thickness of slab are $0.29 a$ and $0.6 a$, respectively, where $a$ is the lattice constant of the PC [see Fig. 1(a)]. The refractive index of the dielectric slab is assumed to be 3.4 , which corresponds to that of silicon at $1.55 \mu \mathrm{m}$. In Table I, we show the results calculated for the four types of donor defects. In the calculations, the defects are surrounded by more than 12 periods of PC layers. (We have confirmed that the $Q$ factors determined by the in-plane losses through the PC layers are as much as about 2000 000.) In Table I we also show the results for an isolated acceptor-type defect with a typical air hole radius $(0.56 a)$, which we refer to as A1 in the following, and also the transmitting region of a line defect waveguide with one missing row, for comparison. In Table I, we can see that the $Q$ factors of the donor defects (except for L1) become larger than that of the acceptor one (900) and that of L3 is the highest (5200). As for the resonant frequencies of the defects, it is seen that the frequency of L3 is within the transmitting region of the waveguide, which is very important for the device operation as can be easily understood from the operation principle. ${ }^{3}$ (We can also adjust the transmitting region by changing the parameter of the waveguide so as to overlap with the defect frequency. ${ }^{13}$ ) Moreover, we found that the higher order modes of L3 are outside the transmitting region of the waveguide (the next higher mode frequency is $0.285 c / a$ ), which indicates that the defect has a Downloaded 06 Mar 2008 to 130.54.110.22. Redistribution subject

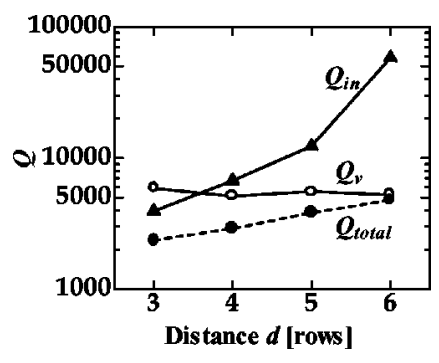

FIG. 2. Plot of the total $(Q)$, in-plane $\left(Q_{\text {in }}\right)$, and vertical $\left(Q_{\mathrm{v}}\right)$ quality factors calculated for L3 donor type defect as a function of the distance $d$ between the defect and waveguide.

wide free spectral range. The results suggest that L3 is the most useful from the viewpoint of the filtering resolution of the device.

Next, we calculated the characteristics of the add/drop device which consists of an L3 defect and a waveguide with one missing row of air holes by using the 3D FDTD method. Figure 2 shows the in-plane $\left(Q_{\text {in }}\right)$, vertical $\left(Q_{\mathrm{v}}\right)$, and total $(Q)$ quality factors of the defect cavity as a function of the distance $(d)$ between the centers of the defect and waveguide [see Fig. 1(a)]. The boundary for separation of vertical from lateral loss (i.e., $Q_{\mathrm{v}}$ from $Q_{\text {in }}$ ) is positioned at a distance of $a$ from the surface of the membrane. In this calculation, $d$ is changed from 3 to 6 rows, where one row is equal to a distance of $\sqrt{3} a / 2$. In Fig. 2, we see that $Q_{\text {in }}$ increases exponentially with the increase of $d$, while $Q_{\mathrm{v}}$ is almost constant. $Q_{\text {in }}$ becomes considerably close to $Q_{\mathrm{v}}$ when $d$ is equal to four rows, and the output efficiency of the device becomes maximal at this distance, as previously described. The efficiency in this case was estimated to be $49.0 \%$ by using the coupled mode theory. ${ }^{3,10}$ The total $Q$ factor at this distance is about 2900, and the filtering resolution of the device [measured by the full width at half maximum (FWHM)] becomes as narrow as $0.5 \mathrm{~nm}$ (assuming that the center wavelength is $1.55 \mu \mathrm{m})$. We also calculated the relationship between the defect frequency and $d$, which demonstrates the fact that the resonant frequency is almost constant $(0.267-0.268 c / a)$, and is within the transmitting region of the waveguide. The result also indicates that the interaction between the waveguide mode and defect mode is not very strong, which ensures that both modes can be designed individually.

Moreover, we investigated the spatial and polarization modes of light emitted from the devices. First, we calculated the spatial mode, in other words, the radiation pattern of the emitted light by assuming an observation plane which distance from the slab center is $4.3 a$. The distribution of the vertical elements of the pointing vectors at this plane was extracted from the electromagnetic field and time averaged for several tens of cycles. The result calculated for the device with an L3 defect is shown in Fig. 3(a). The calculation was also carried out for the device with an A1 (acceptor) defect for comparison [Fig. 3(b)]. In Figs. 3(a) and 3(b), we see that the radiation pattern out of the device with an L3 defect is much narrower than that out of the device with an A1 defect. It is also seen that the radiation pattern from L3 has a peak at the center, while that from A1 is ring-like. For the quantitative evaluation, we have evaluated the radiation angle in the cross section, which is vertical to the slab and parallel to the waveguide. Here, the radiation angle is defined as an apex to AIP license or copyright; see http://apl.aip.org/apl/copyright.jsp 

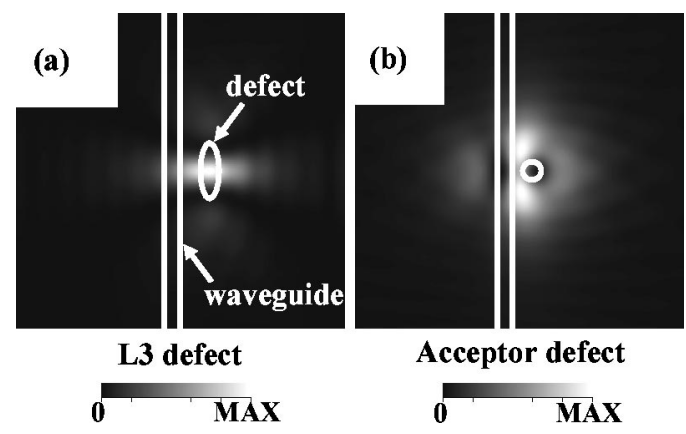

FIG. 3. Calculated radiation patterns of the filtering devices which make use of the L3 defect (a) and the A1 (acceptor) defect (b).

angle of the arc that includes half of the radiation power, which is integrated in the direction vertical to the waveguide. The radiation angle in this cross section is very important for considering crosstalk with the other point defects that will be created along the waveguide in the multichannel devices (the final target). It was found that the radiation angle of the L3 device is as narrow as $30^{\circ}$, while that of the A1 device is as wide as $103^{\circ}$. The results suggest that L3 is superior to the acceptor defects from the viewpoint of crosstalk, as well.

Next, we examined the polarization mode of the emitted light by evaluating its Stokes parameter. Here we defined that the origin of the polarization angle is the direction parallel to a waveguide. The results are as follows: The Stokes parameter $(S 1, S 2, S 3)$ is $(-0.98,-0.00,0.00)$ in the case of the defect L3, and $(0.08,0.00,0.00)$ in the case of the acceptor defect. This clearly shows that the light emitted from the defect L3 is almost linearly polarized in the direction vertical to the waveguide, while that from the defect A1 is considerably depolarized. Both polarization characteristics are useful depending on the characteristics of the external optics and devices, and linearly polarized light from L3 is considered especially useful for most applications.

We will now discuss the reason why the light emitted from the defect L3 is linearly polarized. In general, the far field pattern can be evaluated by the spatial Fourier transformation of a near field pattern. Figures 4(a) and 4(b) show the

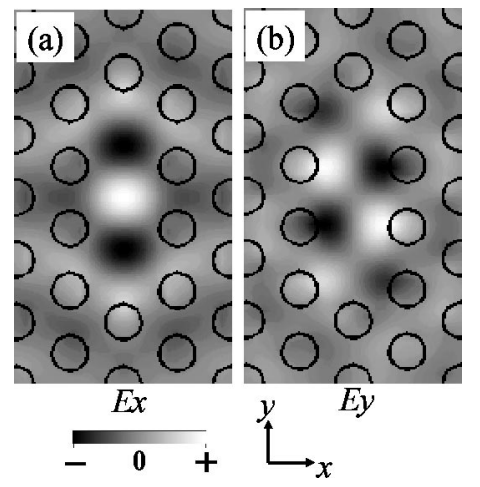

FIG. 4. Electric field distribution of the defect mode of L3. (a) Electric field component along $x$ direction. (b) Electric field component along $y$ direction. The definition of $x$ and $y$ directions is given below the figure. in-plane electric field components $\left(E_{x}\right.$ and $E_{y}$ defined in the figure) of the defect mode, which is observed at the center of the slab. Here, we discuss for simplicity only the light emitted in the direction vertical to the slab. In this case, the DC component of the Fourier transformation, in other words, a simple spatial integration of the near field determines the polarization mode of the radiation. In the figures, we see that $E_{x}$ is symmetric about both $x$ and $y$ axes, while $E_{y}$ is antisymmetric about both axes. Therefore, spatial integration of the $E_{y}$ component inevitably becomes zero while that of $E_{x}$ is not always zero, in fact, the calculated result is not zero. As a result, the light emitted vertically from the defect has the $E_{x}$ component only, which means that the light is linearly polarized in the $x$ direction. The result suggests that the defect engineering with respect to the radiation mode may be possible by developing the above considerations.

In summary, we have theoretically investigated the characteristics of donor defects with one to three missing air holes for the application to surface-emitting channel add/ drop filtering devices. The device which utilizes a lineshaped defect with three missing air holes has been shown to have very high filtering resolution, of which quality factor is as high as 2900 (which corresponds to FWHM of $0.5 \mathrm{~nm}$ assuming that the center wavelength is $1.55 \mu \mathrm{m}$ ). We have also found that the light emitted from this donor-type defect has a very narrow radiation angle of $30^{\circ}$ and is linearly polarized, which are very important for the coupling with external optics. It shows that the utilization of the donor type defect greatly improves the performance of the ultrasmall channel add/drop filtering devices in comparison to that of the previous devices which utilize acceptor type defects. These are very promising and encouraging results, which promote the application of 2D PC slabs, for example, to WDM communication systems.

This work is partly supported by Grant-in-Aid No. 14205012 from the Ministry of Education, Culture, Sports, Science and Technology of Japan and by International Communications Foundation.

${ }^{1}$ S. Noda, A. Chutinan, and M. Imada, Nature (London) 407, 608 (2000).

${ }^{2}$ A. Chutinan and S. Noda, Phys. Rev. B 62, 4488 (2000).

${ }^{3}$ A. Chutinan, M. Mochizuki, M. Imada, and S. Noda, Appl. Phys. Lett. 79, 2690 (2001).

${ }^{4}$ B. S. Song, S. Noda, and T. Asano (unpublished).

${ }^{5}$ O. J. Painter, J. Vuckovic, and A. Scherer, J. Opt. Soc. Am. B 16, 275 (1999).

${ }^{6}$ S. G. Johnson, S. Fan, P. R. Villeneuve, and J. D. Joannopoulos, Phys. Rev. B 60, 5751 (1999).

${ }^{7}$ C. Seassal, Y. Desieres, X. Letartre, C. Grillet, P. Rojo-Romeo, P. Viktorovitch, and T. Benyattou, IEEE J. Quantum Electron. 38, 811 (2002).

${ }^{8}$ J. Vuckovic, M. Loncar, A. Scherer, and H. Mabuchi, Phys. Rev. E 65, 016608 (2001).

${ }^{9}$ H. Y. Ryu, S. H. Kim, H. G. Park, J. K. Hwang, Y. H. Lee, and J. S. Kim, Appl. Phys. Lett. 80, 3883 (2002).

${ }^{10}$ C. Manalatou, M. J. Khan, S. Fan, P. R. Villeneuve, H. A. Haus, and J. D. Joannopoulos, IEEE J. Quantum Electron. 35, 1322 (1999).

${ }^{11}$ K. S. Yee, IEEE Trans. Antennas Propag. AP-14, 302 (1966).

${ }^{12}$ G. Mur, IEEE Trans. Electromagn. Compat. EMC-23, 377 (1981).

${ }^{13}$ M. Notomi, K. Yamada, A. Shinya, J. Takahashi, C. Takahashi, and I. Yokohama, Phys. Rev. Lett. 87, 253902 (2001). 